\title{
RESTAURAÇÕES BIOLÓGICAS COMO ALTERNATIVA PARA REABILITAÇÃO DE DENTES COM EXTENSA DESTRUIÇÃO CORONÁRIA
}

\author{
BIOLOGICAL RESTORATION AS AN ALTERNATIVE TO REHABILITATION \\ OF EXTENSIVELY COMPROMISED TEETH
}

\author{
Ketty Mirelli Vieira VIGNALI'; Caroline Pereira de OLIVEIRA'; Alisson RODRIGUES²; Fernando Freitas PORTELLA; Guilherme \\ Anziliero AROSSI ${ }^{4}$ \\ 1 - Cirurgiã-dentista. Curso de Odontologia, Universidade Luterana do Brasil. Torres, RS, Brasil. \\ 2 - Cirurgião-dentista. Curso de Odontologia, Universidade Luterana do Brasil. Canoas, RS, Brasil. \\ 3 - Cirurgião-dentista. Doutor em Odontologia. Programa de Pós-Graduação em Odontologia, Universidade Luterana do Brasil. Canoas, RS, Brasil. \\ 4 - Cirurgião-dentista. Doutor em Genética. Programa de Pós-Graduação em Promoção da Saúde, Desenvolvimento Humano e Sociedade, \\ Universidade Luterana do Brasil. Canoas, RS, Brasil.
}

\section{RESUMO}

Objetivo: Descrever reabilitações com coroas biológicas por meio de dois casos clínicos, um dente anterior e um posterior. Material e Método: O estudo apresenta dois casos clínicos em que reabilitações de dentes extensamente destruídos foram realizadas. Em um dos casos é apresentada a reabilitação do dente 14 e no seguinte uma reabilitação simultânea dos dentes 21 e 22. Em ambos os casos foram utilizados dentes humanos provenientes de um banco de dentes humanos (BDH). Para a seleção dos dentes, utilizou-se como parâmetros os diâmetros dos dentes a serem reabilitados, bem como as medidas do dente homólogo contralateral. Os dentes obtidos junto a $\mathrm{BDH}$ foram seccionados, a coroa biológica esvaziada e então reembasada com resina composta sobre um modelo de gesso obtido dos pacientes. Após o reembasamento e ajustes oclusais ainda no modelo de gesso, as coroas foram cimentadas com cimento resinoso. Resultados: Em ambos os casos clínicos, os pacientes foram reabilitados adequadamente, de forma rápida e com custo reduzido. Conclusão: Restaurações biológicas podem ser utilizadas para reabilitação de dentes permanentes anteriores e posteriores; além de estimular o correto descarte de futuros dentes extraídos, sendo esses destinados a bancos de dentes humanos.

PALAVRAS-CHAVE: Coroa do Dente; Colagem Dentária; Biomimética.

\section{INTRODUÇÃO}

A odontologia restauradora possui diversas técnicas para restabelecer estética e funcionalmente as estruturas dentárias perdidas em decorrência da doença cárie ou fraturas. Restaurações diretas em resina composta, coroas totais metalocerâmicas, coroas de cerâmica pura, restaurações indiretas do tipo inlays e onlays em resina ou cerâmica, e facetas cerâmicas representam algumas destas técnicas ${ }^{1}$. Em muitas situações o cirurgião-dentista tem como indicação de tratamento mais de uma das opções citadas anteriormente, com longevidade já avaliada por estudos clínicos. Muitas vezes, a escolha do tratamento é realizada considerando questões financeiras, de disponibilidade de tempo do paciente, ou mesmo de questões éticas e filosóficas de cada indivíduo ${ }^{2}$.

Em casos de fratura dentária, além do emprego dos materiais e técnicas tradicionais, há a opção da colagem de fragmento autógeno, com seu primeiro relato na literatura na década de 60, quando se realizou uma reabilitação da fratura num incisivo central superior com o próprio fragmento do dente ${ }^{3,4}$. Além disso, pensando-se na similaridade estrutural dentre os dentes humanos ${ }^{5}$, a colagem de fragmentos homógenos também pode ser uma opção. Na década de 80 , um estudo utilizou dentes extraídos e armazenados em um Banco de Dentes Humanos (BDH), surgindo assim, as colagens homógenas no Brasil. Devido ao sucesso da técnica em dentes anteriores, ela estendeu-se aos dentes posteriores ${ }^{6-8}$.

A técnica de colagem homógena vem sendo tratada na literatura como restaurações biológicas ${ }^{9}$, e constituem uma opção de tratamento que pode ser mais conservadora, rápida, resolutiva e passível de ser aplicada em situações com menores recursos técnicos e financeiros ${ }^{10}$. A técnica consiste em realizar o preparo do remanescente coronário e sua moldagem, e adaptar no modelo de gesso um fragmento dentário. Após a adaptação estar satisfatória, o paciente é chamado para os ajustes finais e a cimentação com um cimento definitivo ${ }^{11}$.

O uso de coroas biológicas para reabilitação, embora não seja muito relatado na literatura, deve ser considerado entre os cirurgiões-dentistas com opção de tratamento em casos específicos, como por exemplo, uso de coroa autógena como provisório para implantes em casos de carga imediata, reabilitação de primeiro molar de pacientes jovens a partir do terceiro molar extraído ${ }^{12}$, reabilitação de dentes decíduos ${ }^{9}$, uso onde não haja acesso a laboratórios de prótese dentária, ou mesmo, o uso como 
alternativa a instalação de materiais artificiais na arcada dentária. Pensando-se na escassez de publicações relatando a técnica, e no aumento do número de profissionais no mercado de trabalho, esse trabalho teve como objetivo instrumentalizar os profissionais para a realização de reabilitações com coroas biológicas. Para isso, foram apresentados dois casos clínicos, um dente anterior e um posterior como exemplos para a descrição da técnica.

\section{RELATO DE CASO}

Dois casos clínicos em que reabilitações foram feitas utilizando-se a técnica de coroas biológicas serão apresentados neste trabalho. Em ambos os casos os elementos cimentados foram obtidos junto a um Banco de Dentes Humanos (BDH). Esse estudo foi aprovado pelo Comitê de Ética em Pesquisa em Seres Humanos da Universidade Luterana do Brasil (ULBRA) sob os pareceres n⿳01. 002.625 e n²28.065, e os pacientes consentiram participação via Termo de Consentimento Livre Esclarecido. Todos os dentes obtidos junto ao banco de dentes passaram por rigoroso processo de descontaminação mecânica e esterilização em autoclave previamente a sua utilização.

\section{Caso I}

Paciente do sexo feminino, 40 anos, leucoderma, apresentou-se ao Projeto de Atendimento Odontológico para Núcleos Especiais e Salas de Espera da ULBRA Campus Torres. Após realizados exames clínicos e radiográficos, o diagnóstico de dente com grande destruição coronária indicava a realização de uma coroa total (Figura 1A). Foram explicadas as opções de tratamento e houve uma tomada de decisão conjunta entre profissional/paciente pela técnica de restauração biológica. A opção da paciente pela reabilitação com coroa biológica se deu por sua condição financeira desfavorável e que por estar recebendo atendimento em uma instituição de ensino com BDH, pôde se valer dessa alternativa de tratamento. Na primeira fase clínica foi realizado o retratamento endodôntico, construção de núcleo de preenchimento em resina composta cor A3 (Charisma, Heraeus Kulzer, Hanau, Alemanha) e preparo para uma coroa total do remanescente dentário com término em ombro arredondado utilizando as pontas diamantadas 2215, 2200, 3203, 3108, 3216, 2215, 4138 (KG Sorensen, Cotia, SP, Brasil) (Figura 1B) e respectivo provisório confeccionado com dente de estoque (Biotone, Dentsply, Petrópolis, RJ, Brasil) e reembasado com resina acrílica JET (Clássico, Campo Limpo Paulista, SP, Brasil). A moldagem de trabalho na arcada superior foi realizada pela técnica do duplo fio, inserindo-se um fio retrator Ultrapak (Ultradent, Indaiatuba, SP, Brasil) mais fino (000) no fundo de sulco e um fio mais espesso (1) sobre o fio mais fino, ambos no dente que foi preparado. A técnica de moldagem realizada foi em dois tempos com moldeiras de estoque lisas (Tecnodente, São Paulo, SP, Brasil), procedendo primeiro a moldagem com silicona de condensação pesada (3M ESPE, Sumaré, SP, Brasil) para posterior realização dos alívios e inserção da silicona de condensação leve (3M ESPE, Sumaré, SP, Brasil). Na arcada inferior, a moldagem foi realizada com alginato Jeltrate (Dentsply, Petrópolis, RJ, Brasil). A escolha da cor foi realizada tendo como parâmetro o dente homólogo, sendo escolhida a cor A3, para isso foi utilizada a escala de cores VITA (Vita Zahnfabrik, Bad Säckingen, Alemanha). Após a escolha da cor a paciente foi liberada.
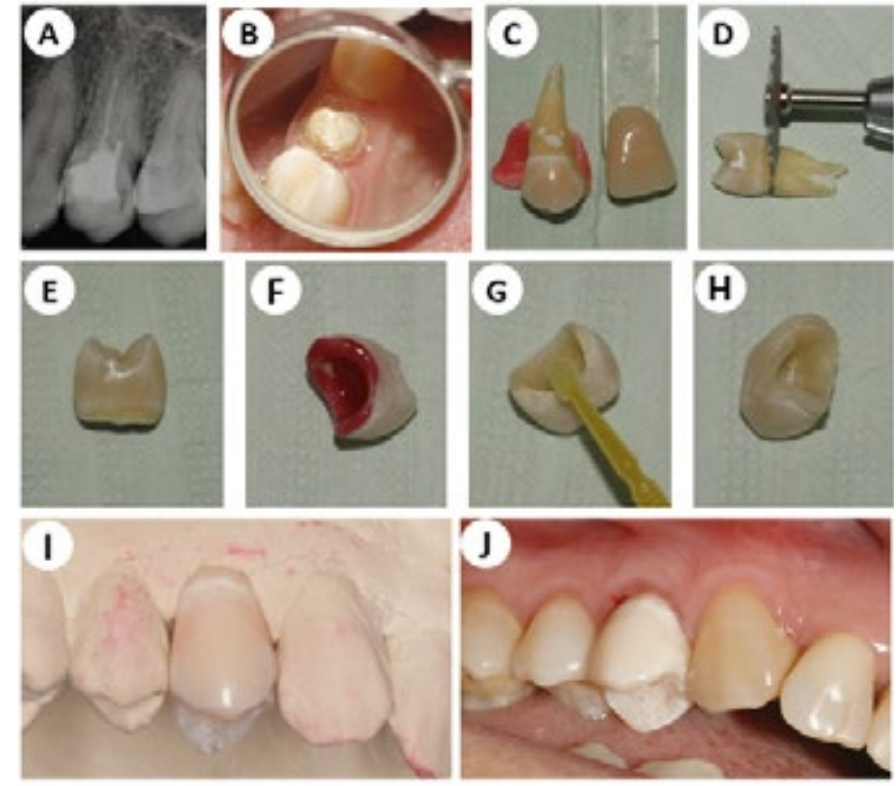

Figura 1 - (A) Radiografia inicial do dente 14, onde pode-se perceber a extensa destruição coronária e necessidade de retratamento endodôntico. (B) Preparo protético do remanescente dentário. (C) Escolha e preparo da coroa biológica; escolha da cor a partir de uma escala Vita. (D) Remoção da raiz. (E) Coroa biológica pronta para receber o esvaziamento da câmara pulpar. (F) Teste, com tinta hidrossolúvel, da adaptação da coroa ao modelo de gesso. (G) Reembasamento da coroa biológica com sistema adesivo e resina composta. $(\mathrm{H})$ Coroa biológica reembasada com resina composta. (I) Adaptação da coroa biológica no modelo de gesso. (J) Resultado após a cimentação.

Com a obtenção do modelo de gesso (Dentsply, Petrópolis, RJ, Brasil), procedeu-se fase laboratorial, na qual o dente foi escolhido no BDH da ULBRA Campus Torres. A escolha do dente se deu com base nos diâmetros mesio-distal, cérvico-oclusal e vestíbulo-lingual do dente perdido. Para obtenção destas medidas teve-se como parâmetro o dente homólogo (dente 24) que foi medido com um compasso de ponta seca (MK Life, Porto Alegre, RS, Brasil) e o espaço existente na área a ser restaurada. Foi levado em consideração também a cor e a forma, até encontrar um dente que se adequou aos critérios mencionados (Figura 1C). No dente selecionado foi realizado um preparo com o corte da raiz $1 \mathrm{~mm}$ aquém da junção cemento-esmalte utilizando disco diamantado (Microdont, São Paulo, SP, Brasil) (Figura 1D) e o esvaziamento da câmara pulpar com broca esférica 1014 (KG Sorensen, Cotia, SP, Brasil) até a obtenção da forma aproximada do preparo (Figura 1E). No modelo, foi feita a adaptação do dente preparado ao troquel por meio da checagem dos pontos de contato com os dentes adjacentes e dos ajustes internos na coroa pela demarcação com tinta hidrossolúvel (Liquid Paper, Newell Rubbermaid Inc., Atlanta, GA, EUA) na área correspondente ao preparo no modelo de gesso (Figura 1F). Essa etapa facilitou a visualização dos pontos do fragmento que necessitavam de desgaste. Para uma melhor adaptação cervical ao término do preparo, foi realizado um reembasamento da coroa oca de esmalte com protocolo adesivo Adper Single Bond 2 (3M ESPE, Sumaré, $\mathrm{SP}$, Brasil) (Figura 1G) e resina composta microhíbrida da cor A3 (Charisma, Heraeus Kulzer, Hanau, Alemanha) (Figura 1H) sobre o troquel de gesso, possibilitando que o fragmento ficasse totalmente justaposto ao preparo (Figura 1I). 
Na segunda fase clínica, a coroa foi testada em boca adaptando-se os pontos de contato até o completo assentamento da coroa no preparo. O ajuste oclusal foi checado com papel carbono (AccuFilm, Parkell Inc., Edgewood, NY, EUA) em máxima intercuspidação habitual (MIH) e nos movimentos excêntricos da mandíbula, protusão e lateralidade, com pontas diamantadas 3118, 1190, 1931, (KG Sorensen, Cotia, SP, Brasil) nos pontos que tiveram uma marcação mais forte, até que se obtivesse uma marcação uniforme nos dentes adjacentes. A adaptação cervical foi testada com sonda exploradora número 5 (Golgran, São Caetano do Sul, SP, Brasil) em movimento de supressão, partindo do sulco gengival, em direção coronal. A cimentação da coroa foi executada após profilaxia e isolamento relativo do campo operatório, utilizando afastadores labiais (FGM Produtos Odontológicos, Joinville, SC, Brasil), fio retrator Ultrapak (Ultradent, Indaiatuba, SP, Brasil) e roletes de algodão. $\mathrm{O}$ condicionamento com ácido fosfórico á 37\% Atacktec (Caithec, São José dos Pinhais, PR, Brasil) foi realizado no fragmento por 30 segundos e no preparo dentário por 15 segundos, sendo estes lavados e secos por 30 segundos. O sistema adesivo foi aplicado numa camada fina no preparo e no fragmento e fotopolimerizado por 20 segundos. O cimento resinoso dual (RelyX ARC, 3M ESPE, Sumaré, $\mathrm{SP}$, Brasil) foi manipulado de acordo com as recomendações do fabricante, inserido no interior da coroa e posicionado sobre o preparo dental até o completo assentamento, e fotopolimerizado por 60 segundos em cada face da coroa biológica, com aparelho led de potência $1250 \mathrm{~mW} / \mathrm{cm} 2$, comprimento de onda de 420$480 \mathrm{~nm}$, gerada por led, frequência de $50 / 60 \mathrm{~Hz}$, (Schuster, Santa Maria, RS, Brasil). Em seguida a oclusão do paciente foi checada novamente com papel carbono e ajustada com o uso de brocas 1014F, 3118F (KG Sorensen, Cotia, SP, Brasil). O acabamento e polimento foram dados com discos de lixa (Praxis, TDV, Pomerode, SC, Brasil) e taça de borracha (Microdont, São Paulo, SP, Brasil) com pasta para polimento (Poligloss, TDV, Pomerode, SC, Brasil). O exame radiográfico foi realizado para registrar a fase final do procedimento.

A coroa biológica cimentada mostrou resultado bastante satisfatório (Figura 1J), em que se restabeleceu o elemento dentário, devolvendo funcionalidade e estética ao paciente. $\mathrm{O}$ caso foi acompanhado a cada dois meses num prazo de seis meses, analisando qualquer deslocamento da restauração, fraturas, alteração dos tecidos periapicais, recidiva de cárie, ausência de doença periodontal e estética. Após dois meses, a cor do remanescente e fragmento estava muito próxima, e ao exame clínico o procedimento restaurador mostrou-se bem adaptado e sem nenhum tipo de alteração, não apresentando também alterações radiográficas.

\section{Caso II}

Paciente de 44 anos, sexo masculino, apresentou-se na clínica de odontologia da ULBRA Campus Torres com grande destruição coronária envolvendo os dentes 21 e 22 (Figura 2A e 2B). Durante o exame clínico, verificou-se boa saúde periodontal, ausência de hábitos parafuncionais, desarmonia do sorriso, alteração cromática nos dentes anteriores e insatisfação com a aparência. $\mathrm{O}$ planejamento reabilitador incluiu duas coroas totais nos dentes 21 e 22, e depois de explicar as vantagens, desvantagens, riscos e benefícios, o paciente manifestou interesse pela reabilitação

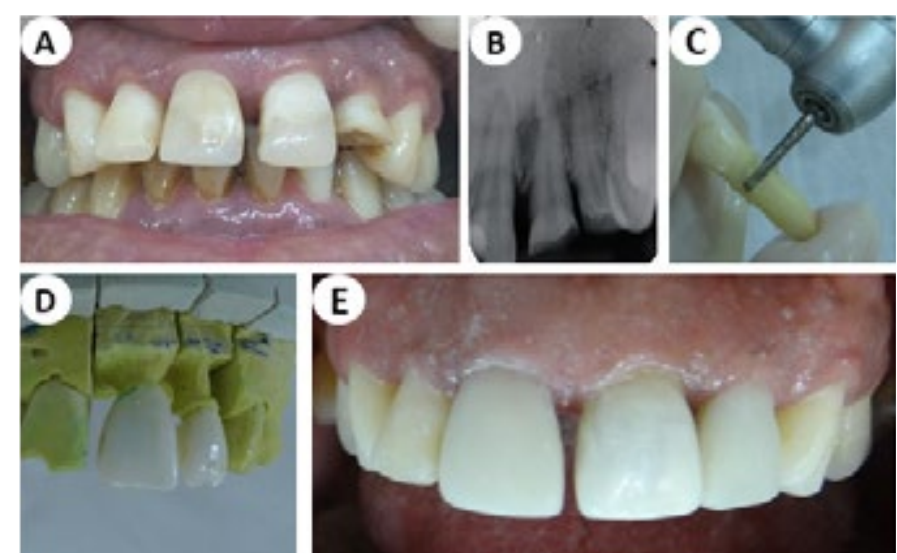

Figura 2 - Reabilitação dos dentes 21 e 22 com coroas biológicas. (A) Aspecto clínico inicial. (B) Radiografia inicial dos dentes 21 e 22. (C) Remoção da raiz na junção amelocementária. (D) Adaptação das coroas aos troqueis no modelo de gesso. (E) Aspecto clínico final.

com a técnica de restauração biológica. A escolha do paciente se deu em função da sua limitação financeira, como também de seu interesse em realizar a reabilitação de uma forma rápida, em poucas consultas.

Para execução do caso foi realizada a primeira fase clínica, que iniciou com o tratamento endodôntico. Para a reabilitação dentária, foi realizada a desobturação de cada canal radicular com brocas Largo Peeso (Maillefer, Dentsply, Petrópolis, RJ. Brasil). Retentores intrarradiculares metálicos pré-fabricados (Angelus, Londrina, PR, Brasil) foram cimentados com cimento resinoso dual (3M ESPE, Sumaré, Brasil), e núcleos de preenchimento confeccionados em resina composta microhíbrida na cor B3 (Charisma, Heraeus Kulzer, Hanau, Alemanha). Para preparo de coroa total foram utilizadas as pontas diamantadas 2215, 2200, 3203, 3108, 3216, 2215, 4138 (KG Sorensen, Cotia, SP, Brasil) e provisório utilizando dente de estoque (Biotone, Dentsply, Petrópolis, RJ. Brasil) e resina acrílica autopolimerizável na cor nº69 (Vipi, São Paulo, SP, Brasil).

Foi realizada moldagem de trabalho do arco superior, pela técnica do duplo fio, inserindo-se um fio retrator (Ultrapak, Ultradent, Indaiatuba, SP, Brasil) mais fino (000) e um fio retrator mais espesso (0) sobre ele promovendo o afastamento gengival e consequente exposição do limite do término cervical. A moldagem foi realizada com silicona de condensação pesada e leve (Vigodent, RJ, Brasil) levadas em boca ao mesmo tempo na arcada superior. Em seguida, foi realizada moldagem do arco antagonista com alginato (Zhermack, RO, Itália) e a escolha da cor, que foi a B3 seguindo a escala de cores VITA (HeraeusKulzer, Hanau, Alemanha).

Com a obtenção do troquel de gesso (Dentsply, Petrópolis, RJ, Brasil), foi realizada a fase laboratorial, com a escolha dos dentes no BDH da ULBRA Campus Torres a partir dos diâmetros do dente 21 e dente 22, considerando cor e forma necessárias ao paciente. Os dentes foram autoclavados a $121^{\circ} \mathrm{C}$ durante 40 minutos e depois mantidos em soro fisiológico. Os dentes escolhidos foram seccionados acima do limite amelocementário com ponta diamantada 2135 (KG Sorensen, Cotia, SP, Brasil) (Figura 2C) e esvaziamento da câmara pulpar com ponta diamantada esférica 1014 (KG Sorensen, Cotia, SP, Brasil) até obtenção da 
forma aproximada do preparo; foi realizada a checagem dos pontos de contato e ajustes com a demarcação através de papel carbono (AccuFilm, Parkell Inc., Edgewood, NY, EUA) na área correspondente ao preparo no troquel de gesso, o que facilitou a visualização dos pontos do fragmento que necessitavam de desgaste. Para uma melhor adaptação cervical ao término do preparo, realizou-se reembasamento das coroas ocas de esmalte com protocolo adesivo (3M ESPE, Sumaré, SP, Brasil) e resina composta micro híbrida B3 (Charisma, HeraeusKulzer, Hanau, Alemanha) sobre o troquel de gesso (Dentsply, Petrópolis, RJ, Brasil), possibilitando que os fragmentos fiquem totalmente justapostos ao preparo (Figura 2D).

Na segunda fase clínica, as coroas de esmalte foram testadas em boca e o ajuste oclusal checado com papel carbono para articulação (AccuFilm, Parkell Inc., Edgewood, NY, EUA). Houve ajustes com pontas diamantadas 3118, 1190, 1931 (KG Sorensen, Cotia, SP, Brasil) nos locais necessários. A adaptação cervical foi testada com sonda exploradora número 5 (Golgran, São Caetano do Sul, SP, Brasil) em movimento de supressão, partindo do sulco gengival em direção coronal.

As cimentações das coroas biológicas foram executadas após profilaxia e isolamento relativo do campo operatório, condicionamento com ácido fosfórico (Atacktec, Caithec, São José dos Pinhais, PR, Brasil) a $37 \%$ durante 30 segundos no interior das coroas e 30 segundos no preparo dentário, lavagem e secagem pelo mesmo tempo, aplicação do sistema adesivo no preparo e fragmento e fotopolimerização por 20 segundos, com aparelho led de potência $1250 \mathrm{~mW} / \mathrm{cm} 2$, comprimento de onda de 420$480 \mathrm{~nm}$, gerada por led, frequência de $50 / 60 \mathrm{~Hz}$, (Schuster, Santa Maria, RS, Brasil). Usou-se cimento resinoso dual (RelyX ARC, 3M ESPE, Sumaré, SP, Brasil), que foi manipulado de acordo com as orientações do fabricante e introduzido no interior da coroa, posicionado sobre o preparo dentário e polimerizado por 60 segundos em cada face da coroa biológica.

A oclusão foi checada com papel carbono articular e ajustada com o uso de pontas diamantadas de acabamento 1190F, 2135F, 3118F, 3195F, 1190FF, 3168FF, (KG Sorensen, Cotia, SP, Brasil). O polimento foi dado com uso sequencial de discos de lixa (Sof-Lex, 3M ESPE, Sumaré, SP, Brasil) e taça de borracha (Microdont, São Paulo, SP, Brasil) e pasta de polimento de granulação média e fina (Asfer, São Caetano do Sul, SP, Brasil). Exame radiográfico final foi realizado para conferir a adaptação cervical das coroas.

Na semana seguinte, houve fratura obliqua da coroa biológica do dente 22, que foi reabilitado com faceta de resina composta microhíbrida cor B3 (Charisma, Heraeus Kulzer, Hanau, Alemanha). Ao final, obteve-se a reabilitação do sorriso, com adequação da cor com faceta direta em resina composta no dente 11 (Figura 2E). Planejou-se a proservação inicial do caso em um período de dois meses.

\section{DISCUSSÃO}

Os casos clínicos relataram uma técnica alternativa de reabilitação de coroas unitárias com restaurações biológicas, que se mostrou eficaz em restabelecer uma condição oral favorável ao paciente. As técnicas descritas nesse trabalho apresentam como vantagens, em relação a restaurações protéticas convencionais, a capacidade de devolver função, brilho, e desgaste natural para o elemento dental comprometido, especialmente, porque nenhum material odontológico pode inteiramente substituir o tecido dentário ${ }^{13}$.

Estas restaurações possuem uma boa estética, tendo uma performance cromática longitudinal semelhante aos demais dentes, tendo em vista serem restaurações feitas de dentes naturais ${ }^{14}$; e permitem o reestabelecimento dentário com uma melhor reconstrução dos contornos dentais, além de uma lisura de superfície natural, já que esta é proveniente do próprio esmalte dentário ${ }^{11}$. A oclusão em dente natural é mais facilmente restabelecida e mantida, tornando consequentemente estas restaurações mais resistentes ao desgaste. Além disso, o fato de o paciente estar recebendo estrutura dentária como material restaurador pode agir como um fator psicológico positivo.

As indicações para esta técnica são para dentes com grande destruição coronária, seja por cárie ou fraturas que apresentem tecido gengival sadio. As principais contraindicações desse tratamento se dão em dentes com núcleo metálico fundido, preparos com términos cervicais muito abaixo da linha gengival, dentes que apresentam o periodonto comprometido e em molares com destruição parcial da coroa, nos quais o preparo do remanescente coronário não oferece extensão e profundidade suficientes para acomodar um fragmento com espessura razoável ${ }^{11,15-17}$.

Quanto às limitações encontradas para a realização de casos que envolvem restaurações biológicas, tem-se a necessidade de um BDH que ofereça opções de tamanho, cor e forma em livre escolha, para o caso específico de cada paciente; e a não aceitação pelos pacientes de receberem um dente de outrem. A utilização de coroas obtidas junto a um BDH respaldou a proteção biológica do cirurgião-dentista e do paciente. O BDH é uma instituição sem fins lucrativos, vinculado ou não a uma faculdade, tendo por objetivo coletar, limpar e armazenar dentes humanos de acordo com as normas de biossegurança ${ }^{18}$ para posterior cessão destes para realização de pesquisas ou treinamento laboratorial. Sendo o dente um órgão, é necessária a autorização do doador para que se possa utilizá-lo ${ }^{19}$. Após sua obtenção legal, os dentes devem passar por um rigoroso processo de desinfecção, que pode ser realizada a partir de sua imersão química, ou então passar pelo processo de esterilização em autoclave.

Outra limitação é a dificuldade técnica de fazer a adaptação interna da coroa natural no preparo dental. O desgaste interno, feito manualmente com alta rotação e testada repetidamente no modelo de gesso até a adaptação visual, pode gerar fragilizações da estrutura, produzindo ocasionalmente áreas com espessura heterogênea, o que cria regiões mais susceptíveis à fratura. Além disso, a inclinação correta da coroa também depende da sensibilidade e do treinamento do profissional. Uma forma descrita na literatura para contornar essas limitações técnicas é a utilização do sistema CAD/CAM. Nesse sistema, o método de confecção da coroa biológica conta com a inclusão da coroa dentária num bloco pré-utilizado de resina CAD/CAM que é usinado, gerando uma coroa perfeitamente adaptada ao preparo e com uma espessura homogênea controlada digitalmente ${ }^{12}$.

Neste trabalho pôde-se observar uma dificuldade para encontrar dentes anteriores superiores íntegros, uma vez que estes, na maioria das vezes, são extraídos por extensa destruição coronária. Bem como dentes com as dimensões e cor que possibilitem um ajuste satisfatório, concordando com relatos na literatura que 
afirmaram que esta técnica tem a seleção de dentes como a maior dificuldade $\mathrm{e}^{14,20,21}$. É importante salientar que o acompanhamento do paciente deve ser feito abordando aspectos como conscientização sobre higiene bucal, pois pelo fato de o material restaurador aplicado no tratamento ser tecido dentário a restauração pode ser colonizada por placa bacteriana, e com seu acúmulo, lesões de cárie podem se desenvolver ${ }^{11}$.

A metodologia empregada no procedimento permite a recuperação do elemento dental, que provavelmente estaria condenado à reabilitação protética. A partir da execução do procedimento restaurador biológico e de achados na literatura ${ }^{14}$, esta técnica mostrou-se viável devido à facilidade de se aplicar na clínica odontológica, desde que sejam seguidos os protocolos necessários para sua execução. A técnica não demanda equipamentos especiais nem processos complexos para sua execução. Alternativas restauradoras são importantes para que nossos pacientes disponham de opções clínicas de qualidade e baixo custo, à medida que reabilitações se tornem imprescindíveis ${ }^{22}$.

\section{CONCLUSÃO}

Restaurações biológicas podem ser utilizadas em restaurações de dentes permanentes anteriores e posteriores; além de estimular o correto descarte de futuros dentes extraídos, sendo esses destinados a bancos de dentes humanos.

\section{REFERÊNCIAS}

01. Alves Neto A, Albernaz A, Almeida Jr E, Soares E, Rocha, MCS. Restaurações biológicas em dentes decíduos: revisão e relato de caso clínico. Rev ABO Nac. 2003; 11(4): 233-8.

02. Opdam NJM, Frankenberger R, Magne P. From 'Direct Versus Indirect' Toward an Integrated Restorative Concept in the Posterior Dentition. Operative Dent. 2016; 41(7): S27-34.

03. Busato ALS. Colagem autógena e heterógena de dentes anteriores fraturados. RGO. 1986; 13(10): 16-23.

04. Chosak A, Eidelman E. Rehabilitation of a fractured incisor using the patients natural crown. Case report. J Dent Child. 1964; 31(1): 519-21.

05. Cabrera CAG, Pinzan A, Castro Cabrera M, Henriques JFC, Janson G, Freitas MR. Estudo biométrico em dentes de humanos. Dental Press J Orthod. 2011; 16(4): 111-22.

06. Imparato JCP, Bonecker MJS, Duarte DA, Pinto ACG. Restauração de dentes decíduos anteriores: técnica alternativa de colagem de coroas naturais. J Bras Odontopediatr Odontol Bebê. 1998; 1(1): 63-72.

07. Gabrielli F, Dinelli W, Fontana UF, Porto CLA. Apresentação e avaliação de uma técnica de restauração de dentes anteriores com fragmentos adaptados de dentes extraídos. RGO. 1981; 29(2): 83-7.

08. Baratieri LN, Ritter AV, Monteiro Jr S, Mello Filho JC. Tooth fragment reattachment: an alternative for restoration of fractured anterior teeth. Pract Periodonts Aesthet Dent.1998; 10(1): 115-25.

09. Sanches K, Carvalho FKD, Nelson-Filho P, Assed S, Silva FWGDP, Queiroz AMD. Biological restorations as a treatment option for primary molars with extensive coronal destruction: report of two cases. Braz Dent J. 2007; 18(3): 248-52.

10. Marturelli R, Cavalcanti NM, Souza FB, Porto POB, Silva CHV. Alternativa estética para reconstrução de dentes anteriores fraturados. Stomatos. 2007; 13(25): 123-30.

11. Montenegro G, Silva WO, Barth THS. Restaurações biológicas homólogas. J Bras Clin Odontol Int. 2003; 7(41): 383-6.

12. Schlichting LH, Schlichting KK, Stanley K, Magne M, Magne P. An approach to biomimetics: the natural CAD/CAM restoration: a clinical report. The Journal of Prost Dent. 2014; 111(2): 107-15.

13. Tavano KTA, Botelho AM, Motta TP, Paes TMO. “Biological restoration": total crown anterior. Dent traumatol. 2009; 25(5): 535-40.

14. Corrêa-Faria P, Alcântara CEP, Caldas-Diniz MV, Botelho AM, Tavano KTA. "Biological restoration": Root canal and coronal reconstruction. Journal of Esthetic and Restorative Dent. 2010; 22(3): 168-77.

15. González Hernández PA, Busato ALS. Restaurações biológicas com fragmentos dentários. Rev Bras Odontol. 1994; 51(2): 22-8.

16. Costa N, Vargas LT, Ferreira TJ, Rabacov PT, Ferreira EFM. Colagem homógena em dentes permanentes. RGO. 2001; 49(3): 151-4.

17. Graner ROM, Imparato JCP. Restaurações biológicas em dentes decíduos. Colagem de fragmentos de dentes naturais. São Paulo: Editora Santos; 2005.

18. Imparato JCP, Duarte DA, Guedes-Pinto AC. Banco de Dentes: importância e valorização do órgão dentário. J Assoc Paul Cir Dent. 1997; 51(6): 19.

19. Gomes GM, Mendes Y, Mongruel OS, Leide M, Schmidt V, Kozlowski AJ. Utilização de dentes humanos: aspectos éticos e legais. RGO. 2013; 61(s0): 477-83.

20. Busato ALS, Antunes M. Colagem heterógena em dentes anteriores fraturados. RGO. 1984; 32(2): 137-40.

21. Cuman V, Pereira SK, Restaurações biológicas: uma opção de tratamento para dentes anteriores fraturados. Ci. Biol. Saúde. 2003; 9(1): 41-6.

22. Isaia AMB, Pozzobon RT, Pithan JC, Marchiori J, Pelissari A. Colagem heterógena em dentes posteriores. RGO. 1996; 44(5): 277-9.

\section{ABSTRACT}

Objective: To describe rehabilitations with biological crowns by two clinical cases, one anterior and one posterior. Methods: The study presents two clinical cases in which extensively damage teeth were rehabilitated. In one, the rehabilitation of tooth 14 is presented and, in the other, a simultaneous rehabilitation of teeth 21 and 22 was performed. In both cases, human teeth from a human tooth bank (HTB) were used. For teeth selection, the diameters of the teeth to be rehabilitated, as well as, the measurements of the contralateral homologous tooth were used as parameters. The teeth obtained with HTB were sectioned, the biological crown emptied and then relined with composite resin on cast obtained from the patients. After the reline and occlusal adjustments, the crowns were cemented with resin cement. Results: The patients were rehabilitated properly, quickly and with reduced cost. Conclusion: Biological restorations can be used for rehabilitation of anterior and posterior permanent teeth; besides to stimulate the correct discard of extracted teeth, being these destined to human teeth banks.

KEYWORDS: Tooth Crown; Dental Bonding; Biomimetics. 


\section{AUTOR PARA CORRESPONDÊNCIA}

Guilherme Anzilliero Arossi

Programa de Pós-Graduação em Promoção da Saúde,

Desenvolvimento Humano e Sociedade, Universidade

Luterana do Brasil. Endereço: Avenida Farroupilha 8001,

Canoas, RS, Brasil. Cep. 92425-900.

Telefone: +55 51997188336

E-mail: guilhermeclinica@gmail.com 